\title{
Exciton Dephasing in a Single Carbon Nanotube Studied by Photoluminescence Spectroscopy
}

\author{
Kazunari Matsuda \\ Institute for Chemical Research, Kyoto University, Uji, Kyoto \\ Institute of Advanced Energy, Kyoto University, Uji, Kyoto \\ Japan
}

\section{Introduction}

The lattice vibration strongly affects the dynamics of electrons and excitons in the solid. In bulk semiconductor materials, the loss of coherence (i.e., dephasing) of electrons and excitons occurs by phonon scattering on very fast time scale of sub-picosecond order. The dephasing time and mechanism depend strongly on the dimensionality and size of the electronic states in low-dimensional semiconductors, such as one-dimensional (1D) quantum wires and zero-dimensional quantum dots (Gammon et al., 1996 \& Braun et al., 1997). A single-walled carbon nanotube (SWNT) is a prototypical 1D electronic system and the optical properties of SWNTs have attracted a great deal of attention, both from the perspective of their fundamental physics (Iijima 1991, O'Connell et al., 2002, \& Bachilo et al., 2002), and for their optoelectronic device applications. Potential applications include photoluminescence (PL) (O'Connell et al., 2002 \& Bachilo et al., 2002), electroluminescence (Misewich et al., 2003), nonlinear optical switching media (saturable absorbers) for ultrafast lasers (Rozhin et al., 2006), and fluorescent biolabeling (Leeuw et al., 2007). These optical properties are due to the creation of very stable excitons by enhanced Coulomb interactions of the order of several-hundred meV, even at room temperature (Ando 1997, Kane et al., 2003, Spataru et al., 2004, Wang et al., 2005, \& Maultzsch et al., 2005). The exciton dynamics are dominated by both the exciton dephasing and the energy relaxation processes, in which the exciton-phonon interactions play an important role (Bradley et al., 2007). However, the exciton dephasing time and mechanism in SWNTs is still only poorly understood and under many discussions. Thus, we describe about detail mechanism of the exciton dephasing in a single carbon nanotube.

\section{Optical transition of carbon nanotube}

In 2002, O'Connell reported that the micelle encapsulated semiconducting SWNTs show strong PL signals ( $\mathrm{O}^{\prime}$ Connell et al., 2002). The first observation of PL signals is due to the isolation of individual SWNT preventing from bundling between semiconducting and metallic carbon nanotubes. Lefebvre also fabricated suspended SWNTs between patterned Si pillars and also observed strong PL signals from unprocessed SWNTs (Lefebvre et al., 2003). The black line of Figure 1 shows the typical macroscopic (ensemble averaged) PL 
spectrum from micelle encapsulated SWNTs dispersed in $\mathrm{D}_{2} \mathrm{O}$ solution. The many PL peaks arising from various types of SWNTs with different chirarities (diameters) are observed in the macroscopic PL spectrum. The optically excited e-h pair in SWNTs forms bound hydrogen like exciton state due to strong Coulomb interaction and recombination of exciton (not free e-h pair) is the origin PL signals in the SWNTs. The shape of each PL (or absorption) peak contains physical information including the exciton dynamics. The shape of ensemble averaged PL spectrum shows the near Gaussian function, which means that each SWNT with same chiral indicies is in the different environment (local strain, dielectric constant, and ....). The exciton transition (absorption and PL) of each SWNT is affected from the Gaussian distributed inhomogeneous environment, causes fluctuation of the energy. The PL broadening arising from the inhomogenity is called as "inhomogeneous broadening", which covers the intrinsic optical and electronic properties of SWNTs. In contrast, the effect of the inhomogeneous broadening is eliminated in the PL spectra from a single SWNT (red line in Fig. 1). The sophisticated optical spectroscopic technique, called as a single carbon nanotube spectroscopy, enables us to observe PL spectrum from a single SWNT (Hartschuh et al., 2003, Htoon et al., 2003, Lefebvre et al., 2004, \& Matsuda et al., 2005). The red solid line of Figure 1 shows the PL spectrum from a single SWNT. The PL of a single SWNT shows very clear single peak and very narrow linewidth, which provide us the fruitful information of intrinsic properties of SWNT. The intrinsic linewidth in the PL spectrum of a single SWNT is called as homogeneous linewidth. The homogeneous linewidth contains the dynamical properties of exciton in the SWNT, discussed after in detail.

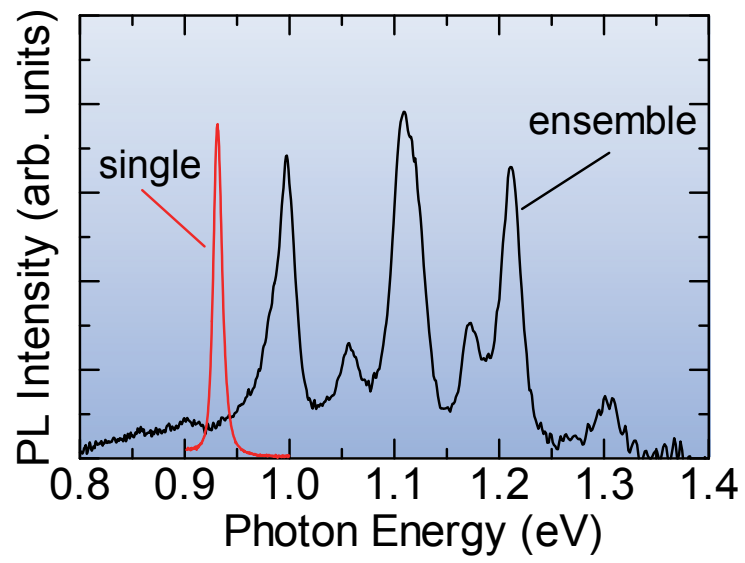

Fig. 1. Ensemble averaged PL spectrum (balck solid line) and PL spectrum of a single SWNT (red line).

\subsection{Homogeneous linewidth broadening of optical spectrum}

In the quantum two-level systems, the optical spectra broaden and have finite linewidths due to finite lifetime of the excited state according to the Heisenberg relationship. There are two kinds of excited state lifetime, energy relaxation time $T_{1}$ and phase relaxation (dephasing) time $T_{2}$. In the molecular systems, the phase relaxation of the excited state 
occurs due to the violation of the excited state such as molecular collisions and so on. In the solid systems, the thermal vibration of lattice, i.e. phonon perturbs the electronic states, leading to the phase relaxation of excited states (dephasing). In contrast, the energy relaxation occurs radiative and nonradiative transition from the excited states to the ground state. The linewidth of the optical spectra, homogeneous broadening $\Gamma$, can be described as,

$$
\Gamma=\hbar\left(1 / T_{1}+2 / T_{2}\right),
$$

where $\hbar(=h / 2 \pi)$ is a Plank constant. Using this relationship, the information on the dephasing time can be obtained from homogeneous broadening of the optical spectra. Also, in the time-domain measurement, the dephasing time can be measured by using nonlinear optical spectroscopy such as photon echo (or four-wave mixing) experiments (Graham et al., 2011).

\section{Experimental technique of single carbon nanotube imaging and spectroscopy}

The samples used for single SWNT spectroscopy were isolated SWNTs synthesized on patterned $\mathrm{Si}$ substrates by an alcohol catalytic chemical vapor deposition method (Maruyama et al., 2002). The Si substrates were patterned with parallel grooves typically from $300 \mathrm{~nm}$ to a few $\mu \mathrm{m}$ in width and $500 \mathrm{~nm}$ in depth using an electron-beam lithography technique. The isolated SWNTs grow from one side toward the opposite side of the groove. We prepared several SWNT samples by changing the growth temperature $\left(650-850{ }^{\circ} \mathrm{C}\right)$ and time (30 sec-10 $\mathrm{min})$. The average number density of isolated SWNTs in the sample is 0.1$1 / \mu \mathrm{m}^{2}$ (Inoue et al., 2006, Matsuda et al., 2008, \& Matsunaga et al., 2008).

Single SWNT PL measurements were carried out from 300 (room temperature) to $5 \mathrm{~K}$ using a home-built variable temperature confocal microscope setup as shown in Fig. 2. The SWNT samples mounted on a stage were excited with a continuous-wave He-Ne laser $(1.959 \mathrm{eV})$ and femtosecond pulsed Ti: $\mathrm{Al}_{2} \mathrm{O}_{3}$ Laser, and the laser beam was focused on the sample

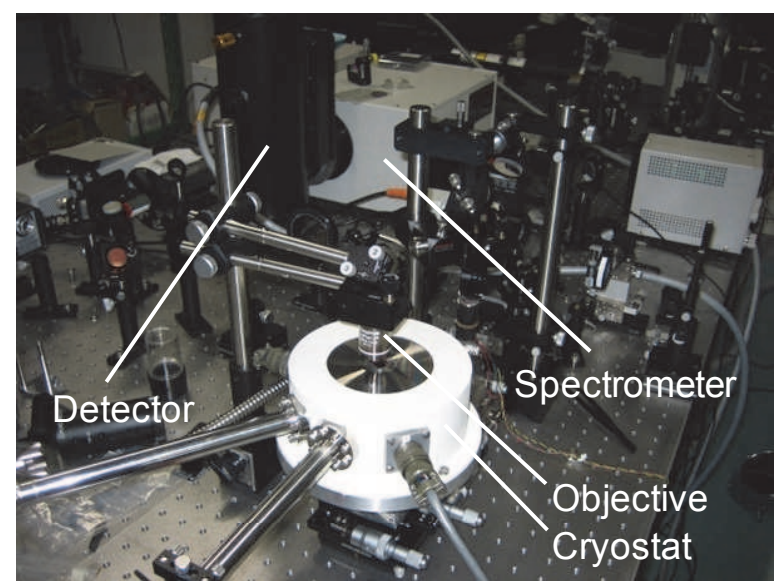

Fig. 2. Picture of experimental setup of low temperature optical microscope for a single SWNT spectroscopy. 
surface through a microscope objective (NA 0.8). The PL signal from SWNTs was spectrally dispersed by a 30 -cm spectrometer equipped with a liquid-nitrogen $\left(\mathrm{LN}_{2}\right)$-cooled InGaAs photodiode array (spectral range: $0.78-1.38 \mathrm{eV}$ ) or a $\mathrm{LN}_{2}$-cooled charge coupled device (spectral range: $\geq 1.20 \mathrm{eV}$ ). The spectral resolution of our system is typically less than 0.7 $\mathrm{meV}$. The detector accumulation time was typically between $30 \mathrm{sec}$ and $1 \mathrm{~min}$. We also obtained PL images of the luminescent SWNTs on the sample, using a Si avalanche photodiode or electron multiple (EM) CCD camera.

Figure 3 shows a typical PL image of isolated semiconducting SWNTs at room temperature. The monitored PL energy range is typically between 1.18 and $1.37 \mathrm{eV}$. Several spatially isolated bright spots can be seen in the 3D image. Each bright spot represents the PL signal from a single luminescent SWNT because the PL spectrum obtained just on each bright spot has only a single PL peak, as shown later. The spot size estimated from the spatial profiles of the PL intensity is about $500 \mathrm{~nm}$.

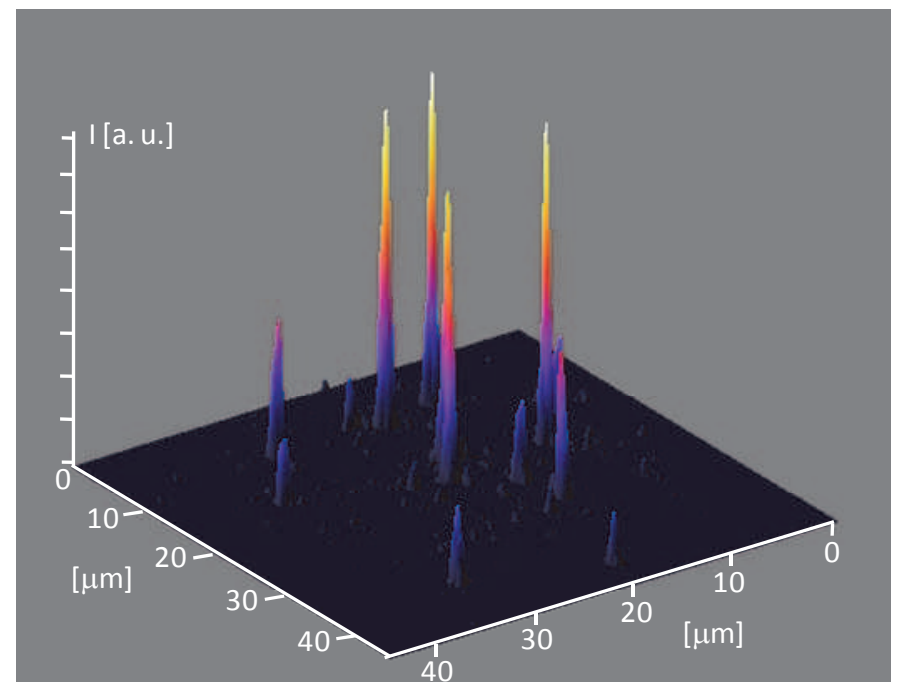

Fig. 3. 3D plots of PL image of single SWNT at room temperature.

\subsection{Single carbon nanotube spectra}

We obtained PL spectra from about 180 different isolated SWNTs with a variety of chiral indices as shown in Fig. 4. The chiral index assignment for each PL spectrum is based on the previous data of the emission energies (Lefebvre, Fraser, et al., 2004). There is a distribution of emission energy ( $\sim$ ten $\mathrm{meV}$ ) even within the same chiral index. Only a single sharp peak can be seen in each spectrum. This is certain evidence that the PL comes from a single SWNT. In addition, the PL linewidth tends to become broader with an increase of the PL peak energy. Figures 5(a) and (b) show, in more details, the typical PL spectra of single isolated SWNTs with relatively small and large diameter [(a): $(10,6), d=0.83 \mathrm{~nm},(\mathrm{~b}):(7,5)$, $d=1.11 \mathrm{~nm}$, ] in the sample.

The red solid lines in Figs. 5(a) and (b) represent single Lorentzian functions and approximately reproduce the experimentally obtained PL spectra. In addition, it is found 


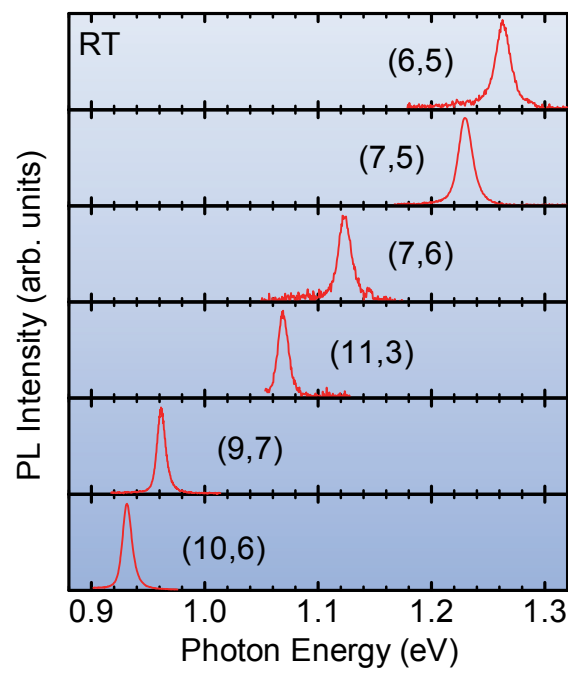

Fig. 4. PL spectra of single SWNT with different chiral index $(n, m)$ (Reprinted with permission from [Inoue et al, 2006]. Copyright, American Physical Society).
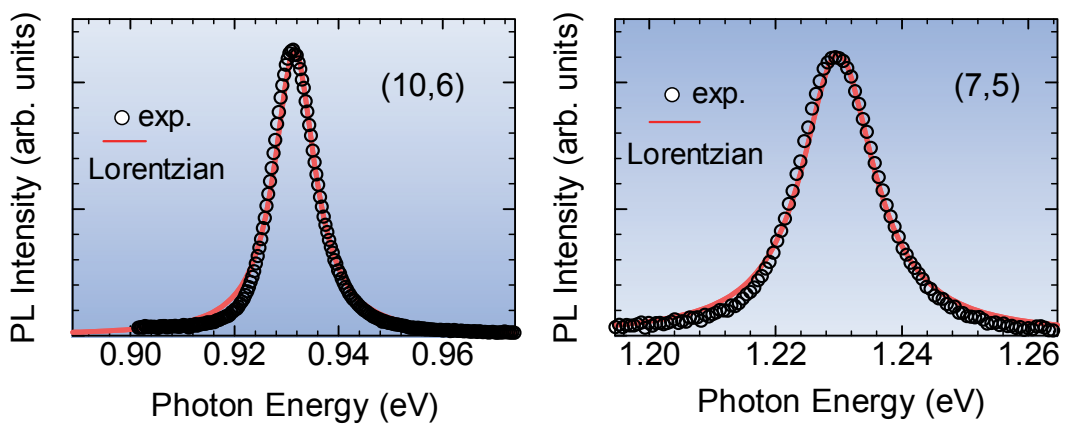

Fig. 5. (a), and (b) PL spectra of single SWNT with chiral index of $(10,6)$ and $(7,5)$ at room temperature (Reprinted with permission from [Inoue et al, 2006]. Copyright, American Physical Society).

that the Stokes shift of SWNTs was very small (less than $5 \mathrm{meV}$ ) for each nanotube species by comparison between absorption and PL spectra of ensemble SWNTs in gelatin matrices. These facts show that the observed PL peaks correspond to the zero-phonon lines of free excitons and the spectral linewidth of the PL spectra is determined by the homogeneous broadening.

\subsection{Dephasing mechanism of exciton state}

Figure 6. shows temperature dependence of PL spectra of single SWNTs from 40 to $297 \mathrm{~K}$. Solid red lines correspond to fitted Lorentzian functions. The SWNT has a chiral index of $(9$, 8 ), based on the emission energies reported (Lefebvre, Fraser, Homma et al., 2004). The PL 
spectral shape can be approximately fitted by a single Lorentzian function at entire temperature range. The linewidths (full-width at half-maximum, FWHM) of the Lorentzian functions are almost dominated by the exciton dephasing time due to exciton-phonon interactions, because the exciton lifetime is longer than $30 \mathrm{ps}$, and the contribution to the linewidth from the exciton lifetime (energy relaxation time) is negligibly small (Hirori et al., 2006, \& Gokus et al., 2008). From the homogeneous linewidth, we calculated the exciton dephasing time at room temperature as about $120 \mathrm{fs}$ for $(9,8)$ nanotubes. The order of the experimentally obtained dephasing time is almost consistent with the Ab initio calculation (Bradley et al., 2007).

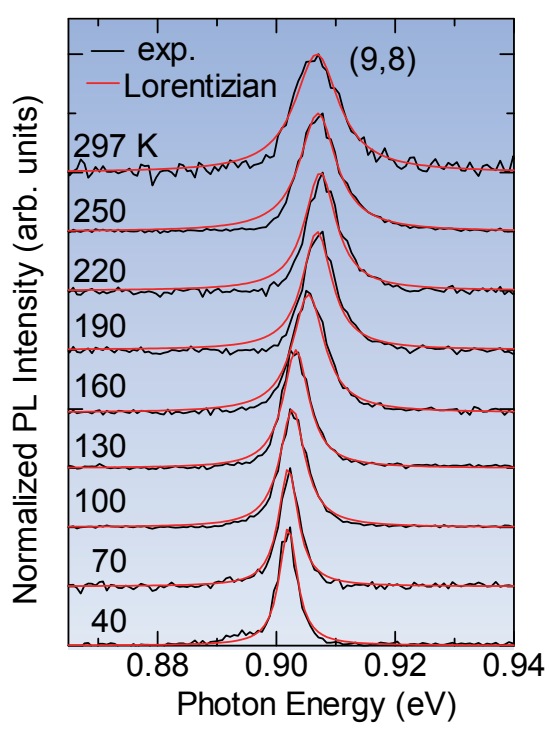

Fig. 6. Temperature dependence of PL spectra of a single SWNT from 40 to $300 \mathrm{~K}$ (Reprinted with permission from [Yoshikawa et al, 2008]. Copyright, American Physical Society).

The PL spectra become narrower when the temperature decreases as shown in Fig. 6. The linewidth of a single SWNT corresponding to a homogeneous linewidth is plotted as a function of temperature in Fig. 7. The temperature dependence of the homogeneous linewidth is usually described as (Karaiskaj et al., 2007):

$$
\Gamma \approx \Gamma_{0}+A \cdot T+\frac{B}{\exp \left(\hbar \omega / k_{B} T\right)-1},
$$

where $\Gamma_{0}$ is the residual linewidth at $T=0 \mathrm{~K}$ including the instrumental spectral resolution, and is the energy of the high frequency phonon modes in SWNTs, such as the radial breathing mode (RBM) and averaged phonon mode. The coefficients $A$ and $B$ are excitonphonon coupling constants for low- and high-frequency modes, respectively. The solid line in Fig. 7 shows the linewidth fitted using Eq. (2). The temperature dependence of the homogeneous linewidth shows almost linear behaviour over a wide temperature range. The value of $A$ obtained for the $(9,8)$ nanotube is $0.020 \mathrm{meV} / \mathrm{K}$. The contribution of the high- 
frequency modes to the linewidth broadening is much smaller than that of the lowfrequency acoustic phonon mode and negligibly small.

We obtained PL spectra at $300 \mathrm{~K}$ from more than 200 different isolated SWNTs with a variety of chiral indices. The homogeneous linewidth as a function of the nanotube diameter $d$ from the PL spectra of single carbon nanotubes is shown in Fig. 8. The smallest values are plotted as the intrinsic linewidth for nanotubes with the same chiral index, because the extrinsic factors (defects, impurities, and so on) cause broadening to a residual linewidth. The PL linewidth at $300 \mathrm{~K}$ clearly increases as the diameter decreases.

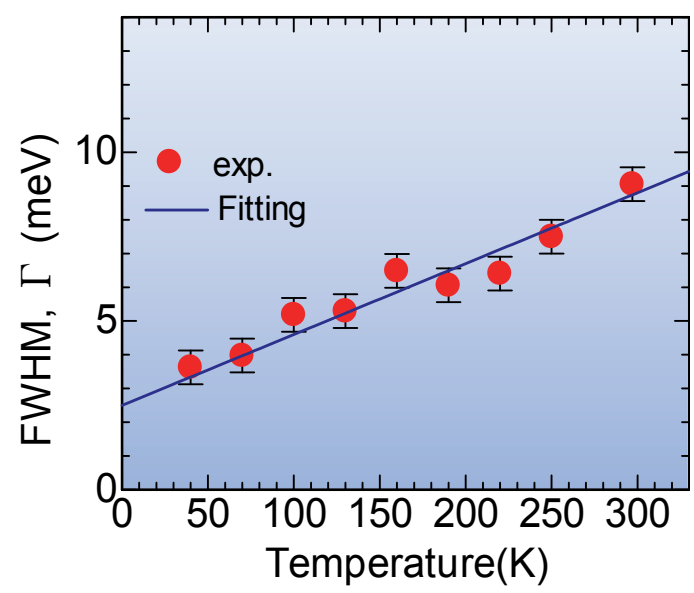

Fig. 7. Temperature dependence of linewidth broadening and theoretically fitted result using Eq. (2) (Reprinted with permission from [Yoshikawa et al, 2008]. Copyright, American Physical Society).

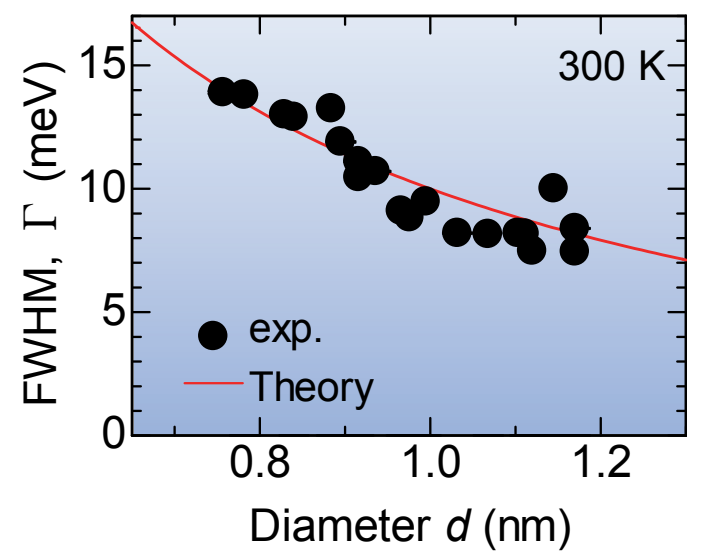

Fig. 8. Diameter dependence of homogeneous linewidth (Reprinted with permission from [Yoshikawa et al, 2008]. Copyright, American Institute of Physics). 
The exciton-phonon coupling constant $A$ for low energy phonon modes is plotted in Fig. 9. The diameter dependence of $A$ is well consistent with that of the homogeneous linewidth at $300 \mathrm{~K}$ in Fig. 8. These observations indicate that the diameter dependence of the PL linewidth observed at $300 \mathrm{~K}$ is not due to the residual linewidth and the nonlinear temperature term in Eq. (2), which is caused by high-frequency modes such as the RBM and averaged phonon mode. From the diameter dependence of the homogeneous linewidth and the exciton-phonon coupling constant $A$, it can be seen that the exciton-phonon interaction is stronger and the exciton dephasing time shorter in smaller tubes. Figure 10 shows the chirality dependence of the homogeneous linewidth at $300 \mathrm{~K}$ and exciton-phonon coupling constant $A$. The data are plotted for SWNTs of around $1.0 \mathrm{~nm}$ indiameter (within the range from 0.9-1.2 nm). Both the homogeneous linewidth and the exciton-phonon coupling constant are almost constant, and neither show the clear chiral angle $\theta$ dependence. However, there is the scattering of the data due to the diameter dependence, as discussed below.

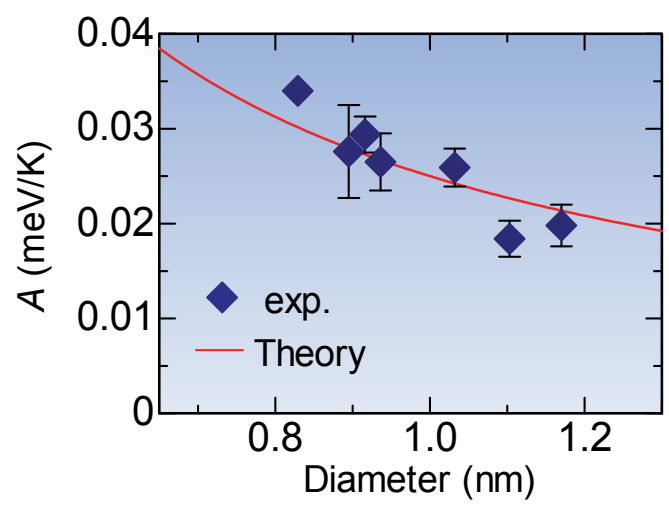

Fig. 9. Diameter dependence of linewidth broadening factor $A$ and theoretically calculated result (Reprinted with permission from [Yoshikawa et al, 2008]. Copyright, American Institute of Physics).

We will discuss the mechanism of the exciton dephasing from the diameter and chirality dependence of the exciton-phonon coupling (homogeneous linewidth) in detail. Here we refer the discussion on the electron-phonon interaction, because there have been no detailed quantitative theoretical studies of exciton-phonon interactions. As described in Jiang et al., 2007, the diameter and chirality dependence of the exciton-phonon interactions show a similar tendency to the electron-phonon interactions (Jiang et al., 2005, \& Jiang et al., 2007). The exciton (electron)-phonon coupling constant using the reduced exciton (electron)phonon matrix element may be written as follows (Suzuura et al., 2002, Jiang et al., 2005, Jiang et al., 2007, \& Popov et al., 2006),

$$
A \propto \frac{\sqrt{M_{0}}}{d} \cdot \sum_{j}\left|g_{j}\right|^{2} E^{-1 / 2},
$$

where $M_{0}$ is the exciton effective mass, $E$ is the exciton energy, and $g_{\mathrm{j}}$ is the reduced matrixelement of the exciton-phonon interaction. As both $M_{0}$ and $E$ are inversely proportional to 
the diameter $d$, the exciton-phonon coupling in Eq. (2) has $1 / d$ dependence. The diameter dependence $(A \propto 1 / d)$ arises from the specific characteristics of the exciton-phonon interaction in $1 \mathrm{D}$ carbon nanotubes.

The linear temperature dependence of the linewidth noted above suggests that the phonon modes that contribute to linewidth broadening are low-energy acoustic modes, such as TW (twisting) and LA (longitudinal acoustic). The chirality dependences of the predicted reduced exciton (electron)-phonon matrix elements for TW and LA phonon modes are described by $\mathrm{g}_{\mathrm{TW}}=\cos 3 \theta$ and $\mathrm{g}_{\mathrm{LA}}=0.66 \sin 3 \theta$, respectively (Suzuura et al., 2002). Using the reduced matrix-elements, we calculated the diameter dependence of the exciton-phonon coupling constant from Eq. (2). The results are indicated by the red solid line in Figs. 8 and 9. The calculated diameter dependence of the homogeneous linewidth from Eqs. (2) and (3) is also shown in Figs. 8 and 9. We assumed that the residual linewidths are constant $(2.0 \mathrm{meV})$ in the theoretically calculated curve, because they are almost constant independent of the diameter, and the variation of the values is small (within $\sim 1 \mathrm{meV}$ ) for each single nanotube. The theoretical calculated line in Fig. 9 agrees closely with the experimental results.

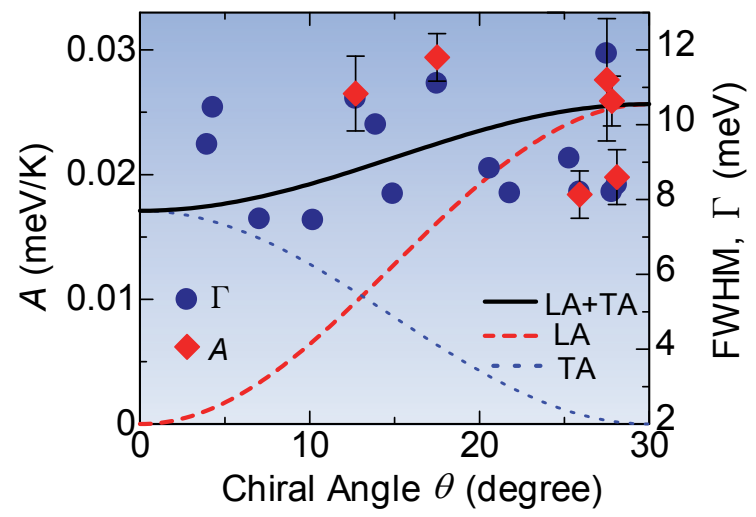

Fig. 10. Chiral angle dependence of linewidth broadening factor $A$ and FWHM at room temperature. (solid line) (Reprinted with permission from [Yoshikawa et al, 2008]. Copyright, American Institute of Physics).

Figure 10 also shows the calculated chirality dependence of the exciton-phonon coupling constant (homogeneous linewidth) for SWNTs of around $1 \mathrm{~nm}$ in diameter, indicated by the solid line. The contributions of the two phonon modes LA and TW are indicated as dashed and dotted lines, respectively. The calculated result indicated by the solid line is in agreement with the experimental results of the chirality dependence of the exciton-phonon coupling constant (homogeneous linewidth). In the small (large) chiral angle nanotubes, the TW (LA) mainly contributes to the exciton-phonon coupling constant (homogeneous linewidth). These observations indicate that both the LA and TA modes contribute to the exciton dephasing for carbon nanotubes.

\subsection{Dephasing exciton state via exciton-exciton interaction}

We studied temperature dependence of the PL spectra of an isolated SWNT excited with a He-Ne laser. The spectra were measured under lower excitation conditions (less than $<100$ 
$\mu W)$. The PL spectra clearly narrow when the temperature decreases (Lefebvre et al., 2004). The exciton energy changes from 5 to $90 \mathrm{~K}(\sim 1 \mathrm{meV})$ is much smaller than that in other compound semiconductor quantum wire ( $12 \mathrm{meV}$ from 5 to $90 \mathrm{~K}$ in GaAs wires) in the weak exciton-phonon coupling regime. This weak temperature dependence is consistent with the experimental fact of narrow PL spectra with Lorentizian lineshape in Fig. 5. Both the dephasing time and the energy relaxation time of the excitons contribute to homogeneous linewidth broadening. The measured PL lifetimes of excitons are considerably longer $(\geq 20$ ps) from 10 to $300 \mathrm{~K}$ (Perebeinos et al., 2005, \& Hirori et al., 2006) and this contribution to the linewidth is negligibly small $(\leq 0.01 \mathrm{meV})$ under lower excitation conditions. Thus, the temperature-dependent linewidths are determined approximately by the dephasing time of the exciton due to the exciton-phonon interactions. Based on the homogeneous linewidth, the exciton-dephasing time is evaluated from $350 \mathrm{fs}$ at $90 \mathrm{~K}$ to more than $940 \mathrm{fs}$ at $5 \mathrm{~K}$.

Figure 11 shows PL spectra obtained from a typical single SWNT [assigned chiral index: (11, $4)]$ at $30 \mathrm{~K}$ and at various excitation intensities of 1.72-eV and 150-fs laser pulses. Each spectrum has a single peak located at $0.941 \mathrm{eV}$. Even in the high-excitation region above about $15 \mathrm{pJ}$ per pulse, the PL spectrum shows a single peak, without a change in the peak energy. Furthermore, additional spectral structures are not observed in any lower energy regions. Similarly, the PL bands due to biexcitons and inelastic exciton scattering are not observed in this spectral region.

Spectrally integrated PL intensities are plotted as a function of excitation laser intensity in Fig. 12. In a low excitation region below $10 \mathrm{pJ}$, the PL intensity grows almost linearly with excitation intensity (as indicated by a dotted line). Conversely, in the higher excitation intensity region (> $20 \mathrm{pJ}$ ), saturation of the PL intensity is clearly apparent. We show normalized PL spectra for a single SWNT, excited with different intensities, on an expanded energy scale in Fig. 13. The spectral linewidth broadens with increasing excitation intensity.

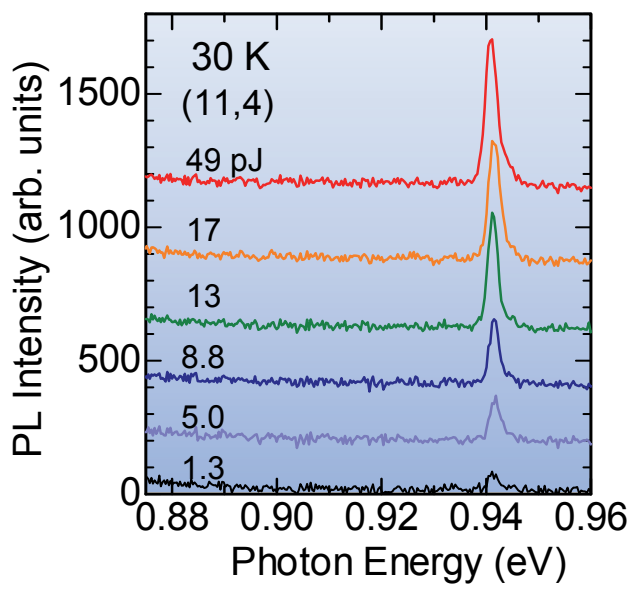

Fig. 11. Excitation power dependence of PL spectra of single SWNT at $30 \mathrm{~K}$ (Reprinted with permission from [Matsuda et al, 2008]. Copyright, American Physical Society).

The homogeneous linewidth is plotted in Fig. 14 as a function of excitation intensity, with the FWHM of the linewidth broadening nonlinearly. This broadening cannot be explained 
by laser heating effects because the nonlinear broadening behavior contradicts the linear temperature dependence of the linewidth broadening [see Fig. 7]. This excitation intensitydependent linewidth broadening indicates that the exciton dynamics are strongly affected by the multiple excitons present in a SWNT.

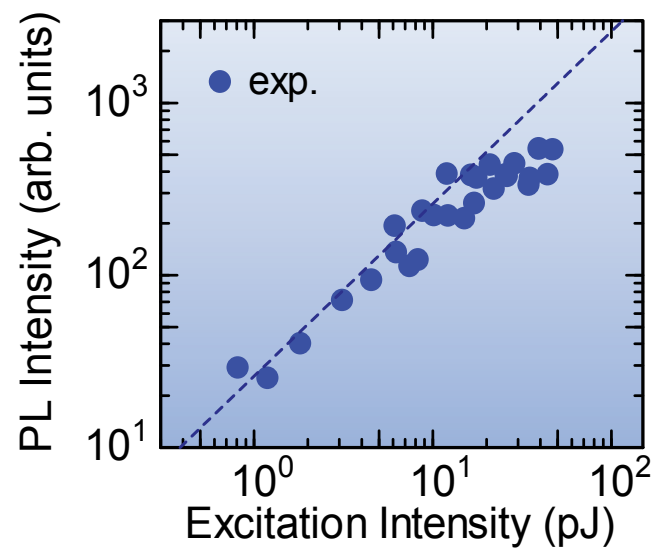

Fig. 12. Integrated PL intensity as a function of excitation intensity. Dotted line corresponds to linear dependence (Reprinted with permission from [Matsuda et al, 2008]. Copyright, American Physical Society).

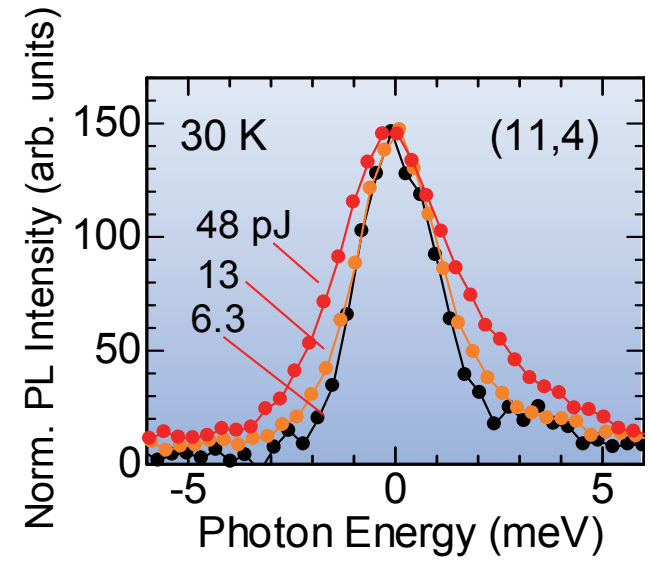

Fig. 13. Normalized PL spectra of a single SWNT on an expanded energy scale excited with different intensities (Reprinted with permission from [Matsuda et al, 2008]. Copyright, American Physical Society).

Under higher excitation conditions, when additional homogeneous linewidth broadening and saturation of the PL intensity occur, more than one exciton is created in a SWNT, leading to a remarkable scattering process between excitons. Exciton-exciton annihilation 
occurs due to the rapid Auger nonradiative recombination process through strong Coulomb interactions (Wang et al., 2004, Ma et al., 2005, Haung et al., 2006, \& Wang et al., 2006).

The PL intensity saturation at higher excitation regions, as shown in Fig. 12, can be explained by the opening of the nonradiative relaxation path due to the exciton-exciton annihilation.

The homogeneous linewidth $\Gamma$ in Fig. 14 is determined by both the excitation power independent exciton-phonon interaction term $\Gamma_{\text {ex-ph }}$ and the excitation power dependent Auger term $\Gamma_{\text {ex-ex, }}$

$$
\Gamma=\Gamma_{e x-p h}+\Gamma_{e x-e x} .
$$

The homogeneous linewidth broadening at higher excitation regions can be explained by the shortening of exciton lifetime due to the Auger process.

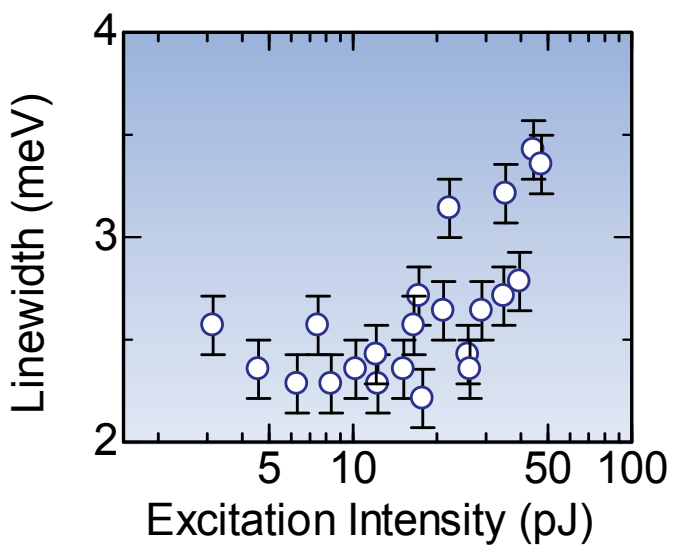

Fig. 14. Homogeneous linewidths as a function of excitation intensity (Reprinted with permission from [Matsuda et al, 2008]. Copyright, American Physical Society).

From the experimental results, the derived exciton-exciton annihilation constant of 1.6 $\mathrm{ps} / \mu \mathrm{m}$ is almost consistent with the theoretically calculated value based on perturbation theory (Wang 2006). From this result, it was found that the multiparticle Auger process occurs very efficiently with an Auger recombination time estimated at $800 \mathrm{fs}$ for $\sim 1 \mu \mathrm{m}$-long SWNT when two excitons are present in a SWNT. This very short, in comparison to bulk semiconductors, time constant is characteristics of low-dimensional systems, and is comparable to the exciton-dephasing time at low temperature. These results indicate that the exciton dephasing is limited under high excitation conditions, not by the exciton-phonon interactions, but by the exciton-exciton interactions.

\section{Conclusion}

In this chapter, we studied the temperature and chirality dependence of the PL linewidth of single carbon nanotubes using single SWNT spectroscopy to clarify the mechanism of exciton dephasing. The PL linewidth of a single carbon nanotube broadened linearly with increasing temperature, indicating that the linewidth and exciton dephasing are determined 
through exciton-phonon interactions. From the chirality dependence of the PL linewidth, we concluded that exciton dephasing is caused by both the longitudinal acoustic and twisting phonon modes. Saturation of the PL intensity and broadening of the PL linewidth in a single SWNT occur simultaneously with an increase in excitation laser intensity. Our findings show that the rapid exciton-exciton annihilation through multiparticle Auger recombination broadens the homogenous linewidth.

\section{Acknowledgment}

The author thanks Y. Miyauchi, Y. Murakami, S. Maruyama, T. Inoue, K. Yoshikawa, R. Matsunaga, and Y. Kanemitsu for collaborations and discussions. This study is partially supported by Grant-in-Aid from MEXT, Japan.

\section{References}

Ando, T. (1997). Excitons in Carbon Nanotubes. Journal of the Physical Society of Japan, Vol. 66, No. 4, pp. 1066-1073.

Bachilo, S. M.; Strano, M. S.; Kittrell, C.; Hauge, R. H.; Smalley, R. E. \& Weisman, R. B. (2002). Structure-assigned Optical Spectra of Single-walled Carbon Nanotubes. Science, Vol. 298, pp. 2361-2366.

Braun, W.; Bayer, M.; Forchel, A.; Zull.; H. Reithmaier J. P.; Filin, A. I.; Walck S. N. \& Reinecke T. L. (1997). Excitonic Wave Packets in $\operatorname{In}_{0.135} \mathrm{Ga}_{0.865}$ As/GaAs Quantum Wires. Physical Review B, Vol. 55, No. 15, pp. 9290-9293.

Bradley, F. H.; Kamisaka, H.; Yamashita K. \& Prezhdo, Oleg V. (2007). Ab Initio Study of Vibrational Dephasing of Electronic Excitations in Semiconducting Carbon Nanotubes. Nano Letters, Vol. 7, No. 11, pp. 3260-3265.

Gammon, D.; Snow, E. S.; Shanabrook, B. V.; Katzer, D. S. \& Park D. (1996). Fine Structure Splitting in the Optical Spectra of Single GaAs Qantum Dots. Physical Review Letters, Vol. 76, No. 16, pp. 3005-3008.

Gokus, T.; Hartschuh, A.; Harutyunyan, H.; Allegrini, M.; Hennrich, F.; Kappes, M.; Green, A. A.; Hersam, M. C.; Araújo, P. T. \& Jorio A. (2008). Exciton Decay Dynamics in Individual Carbon Nanotubes at Room Temperature. Applied Physics Letters, Vol. 92, No. 15, 153116-1-153116-3.

Graham, M. W.; Ma, Y-Z.; Green, A. A.; Hersam, M. C. \& Fleming, G. R. (2011). Pure Optical Dephasing Dynamics in Semiconducting Single-walled Carbon Nanotubes. Journal of Chemical Physics, Vol. 134, No. 3, pp. 034504-1-034504-13.

Hartschuh, A.; Pedrosa, H. N.; Novotny L. \& Krauss, T. D. (2003). Simultaneous Fluorescence and Raman Scattering from Individual Single-walled Carbon Nanotubes. Science, Vol. 301 No. 5638, pp. 1354-1356.

Hirori, H.; Matsuda, K.; Miyauchi Y.; Maruyama S. \& Kanemitsu Y. (2006). Exciton Localization of Single-walled Carbon Nanotubes Revealed by Femtosecond Excitation Correlation Spectroscopy. Physical Review Letters, Vol. 97, No. 25, pp. 257401-1-257401-4.

Htoon, H.; O'Connell, M. J.; Cox, P. J. Doorn, S. K. \& Klimov V. I. (2002). Low Temperature Emission Spectra of Individual Single-walled Carbon Nanotubes: Multiplicity of 
Subspecies within Single-species Nanotube Ensembles. Physical Review Letters, Vol. 93, No. 2, pp. 027401-1-027401-4.

Huang L. \& Krauss, T. D. (2006). Quantized Bimolecular Auger Recombination of Excitons in Single-Walled Carbon Nanotubes. Physical Review Letters, Vol. 96, No. 5, 0574071-057407-4.

Iijima, S. (1991). Helical Microtubules of Graphitic Carbon. Nature, Vol. 354, pp. 56-58.

Jiang, J.; Saito, R.; Gruneis, A.; Chou, S. G.; Samsonidze, G. G.; Jorio, A.; Dresselhaus G. \& Dresselhaus, M. S. (2005). Photoexcited Electron Relaxation Processes in Single-wall Carbon Nanotubes. Physical Review B, Vol. 71, No. 4, 045417-1-045417-9.

Jiang, J.; Saito, R.; Sato, K.; Park, J. S.; Samsonidze, Ge. G.; Jorio, A.; Dresselhaus, G. \& Dresselhaus, M. S. (2007). Exciton-photon, Exciton-phonon Matrix Elements and Resonance Raman Intensity of Single Wall Carbon Nanotubes. Physical Review B, Vol. 75, No. 3, pp. 035405-1-035405-10.

Inoue, T.; Matsuda, K.; Kanemitsu, Y.; Murakami, Y. \& Maruyama S. (2006). Diameter Dependence of Exciton-phonon Interaction in Individual Single-walled Carbon Nanotubes Studied by Microphotoluminescence Spectroscopy. Physical Review B, Vol. 73, No. 23, pp. 233401-1-233401-4.

Kane, C. L., \& Mele, E. J. (2003). Electron Interactions and Scaling Relations for Optical Excitations in Carbon Nanotubes. Physical Review Letters, Vol. 93, No.19, pp. 1974021-197402-4.

Karaiskaj, D., \& Mascarenhas, A. (2007) Role of Electron-phonon Interactions and External Strain on the Electronic Properties of Semiconducting Carbon Nanotubes. Physical Review B, Vol. 75, No. 11, pp. 115426-1-115426-4.

Leeuw, T. K; Reith, R. M.; Simonette R. A.; Harden M. E.; Cherukuri, P.; Tsyboulski, D. A.; Beckingham, K. M. \& Weisman R. B. (2007). Single-walled Carbon Nanotubes in the Intact Organism: Near-IR Imaging and Biocompatibility Studies in Drosophila. Nano Letters, Vol. 7, No. 9, pp. 2650-2654.

Lefebvre, J.; Homma, Y. \& Finnie P. (2003). Bright Band Gap Photoluminescence from Unprocessed Single-walled Carbon Nanotubes. Physical Review Letters, Vol. 93, No. 21, pp. 217401-1-217401-4.

Lefebvre, J.; Fraser, J. M.; Homma, Y. \& Finnie P. (2004). Photoluminescence from Singlewalled Carbon Nanotubes: a Comparison Between Suspended and Micelleencapsulated Nanotubes. Applied Physics A, Vol. 78, No. 8, pp. 1107-1110.

Lefebvre, J.; Fraser, J. M.; Finnie, P. \& Homma, Y. (2004). Photoluminescence from an Individual Single-walled Carbon Nanotube. Physical Review B, Vol. 69, No. 7, pp. 075403-1-075403-5.

Lefebvre, J.; Finnie, P. \& Homma, Y. (2004). Temperature-dependent Photoluminescence from Single-walled Carbon Nanotubes. Physical Review B, Vol. 70, No. 4, 045419-1045419-8.

Ma, Y-Z.; Valkunas, L.; Dexheimer, S. L.; Bachilo, S. M. \& Fleming, G. R. (2005). Femtosecond Spectroscopy of Optical Excitations in Single-Walled Carbon Nanotubes: Evidence for Exciton-Exciton Annihilation. Physical Review Letters, Vol. 94, No. 15, pp. 157402-1-157402-4. 
Maruyama, S.; Kojima, R.; Miyauchi Y.; Chiashi S. \& Kohno M. (2002). Low-temperature Synthesis of High-purity Single-walled Carbon Nanotubes from Alcohol. Chemical Physics Letters, Vol. 360, No. 3-4, pp. 229-234.

Matsuda, K.; Kanemitsu, Y.; Irie, K.; Saiki, T.; Someya, T.; Miyauchi, Y. \& Maruyama, S.; (2005). Photoluminescence Intermittency in an Indivitual Single-walled Carbon Nanotube at Room Temperature. Applied Physics Letters, Vol. 86, No. 12, pp. 1231161-123116-3.

Matsuda, K.; Inoue, T.; Murakami, Y.; Maruyama, S. \& Kanemitsu, Y. (2008). Physical Review B, Vol. 77, No. 19, pp. 193405-1-193405-4.

Matsunaga, R.; Matsuda, K. \& Kanemitsu Y. (2008). Evidence for Dark Excitons in a Single Carbon Nanotube due to the Aharonov-Bohm Effect. Physical Review Letters, Vol. 101, No. 14, pp. 147404-1-147404-4.

Maultzsch, J.; Pomraenke, R.; Reich, S.; Chang, E.; Prezzi, D.; Ruini, A.; Molinari, E.; Strano, M. S.; Thomsen, C. \& Lienau, C. (2005). Two-photon Photoluminescence and Exciton Binding Energies in Single-walled Carbon Nanotubes. Physical Review B, Vol. 72, No. 24, pp. 241402(R)-1-241402(R)-4.

Misewich, J. A.; Martel, R.; Avouris, Ph.; Tsang, J. C.; Heinze, S. \& Tersoff, J. (2003). Electrically Induced Optical Emission from a Carbon Nanotube FET. Science, Vol. 300, No. 5620, pp. 783-786.

O'Connell, M. J.; Bachilo, S. M.; Huffman, C. B.; Moore, V. C.; Strano, M. S.; Haroz, E. H.; Rialon, K. L.; Boul, P. J.; Noon, W. H.; Kittrell, C.; Ma, J.; Hauge, R. H.; Weisman R. B. \& Smalley R. E. (2002). Band Gap Fluorescence from Individual Single-walled Carbon Nanotubes. Science, Vol. 297, No. 5581, pp. 593-596.

Perebeinos, V.; Tersoff, J. \& Avouris, P. (2005). Radiative Lifetime of Excitons in Carbon Nanotubes. Nano Letters, Vol. 5, No. 12, 2495-2499.

Popov V. N. \& Lambin, P. (2006). Intraband Electron-phonon Scattering in Single-walled Carbon Nanotubes. Physical Review B, Vol. 74, No. 7, pp. 075415-1-075415-4.

Rozhin, A. G.; Sakakibara, Y.; Namiki, S.; Tokumoto, M.; Kataura, H. \& Achiba, Y. (2006). Sub- 200-fs Pulsed Erbium-doped Fiber Laser using a Carbon Nanotubepolyvinylalcohol Mode Locker. Applied Physics Letters, Vol. 88, No.5, pp. 051118-105118-3.

Spataru, C. D.; Ismail-Beigi, S.; Benedict, L. X. \& Louie, S. G. (2004). Excitonic Effects and Optical Spectra of Single-Walled Carbon Nanotubes. Physical Review Letters, Vol. 92, No. 7, pp. 077402-1-077402-4.

Suzuura H. \& Ando, T. (2002). Phonons and Electron-phonon Scattering in Carbon Nanotubes. Physical Review B, Vol. 65, No. 23, pp. 235412-1-235412-4.

Wang, F.; Dukovic, G.; Knoesel, E.; Brus, L. E. \& Heinz, T. F. (2004). Observation of Rapid Auger Recombination in Optically Excited Semiconducting Carbon Nanotubes. Physical Review B, Vol. 70, No. 24, pp. 241403(R)-1-241403(R)-4.

Wang, F.; Dukovic, G.; Brus, L. E. \& Heinz T. F. (2005). The Optical Resonances in Carbon Nanotubes Arise from Excitons. Science, Vol. 308, Vol. 5723, pp. 838-841.

Wang, F.; Wu, Y.; Hybertsen, M. S. \& Heinz, T. F. (2006) Auger Recombination of Excitons in One-Dimensional Nanostructures. Physical Review B, Vol. 73, No. 24 pp. 245424-1245424-5. 
Yoshikawa K.; Matsunaga, R; Matsuda, K. \& Kanemitsu, Y. (2009). Mechanism of Exciton Dephasing in a Single Carbon Nanotube Studied by Photoluminescence Spectroscopy. Applied Physics Letters, Vol. 94, No. 9, pp. 093109-1-093109-3. 


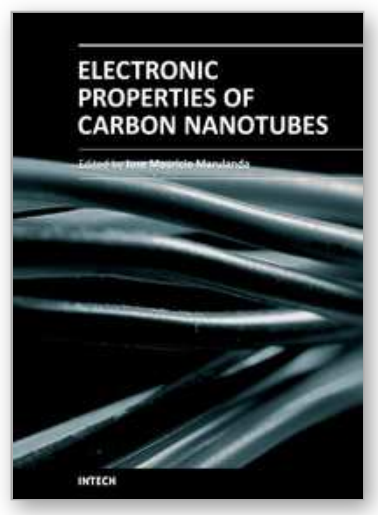

\author{
Electronic Properties of Carbon Nanotubes \\ Edited by Prof. Jose Mauricio Marulanda
}

ISBN 978-953-307-499-3

Hard cover, 680 pages

Publisher InTech

Published online 27, July, 2011

Published in print edition July, 2011

Carbon nanotubes (CNTs), discovered in 1991, have been a subject of intensive research for a wide range of applications. These one-dimensional (1D) graphene sheets rolled into a tubular form have been the target of many researchers around the world. This book concentrates on the semiconductor physics of carbon nanotubes, it brings unique insight into the phenomena encountered in the electronic structure when operating with carbon nanotubes. This book also presents to reader useful information on the fabrication and applications of these outstanding materials. The main objective of this book is to give in-depth understanding of the physics and electronic structure of carbon nanotubes. Readers of this book should have a strong background on physical electronics and semiconductor device physics. This book first discusses fabrication techniques followed by an analysis on the physical properties of carbon nanotubes, including density of states and electronic structures. Ultimately, the book pursues a significant amount of work in the industry applications of carbon nanotubes.

\title{
How to reference
}

In order to correctly reference this scholarly work, feel free to copy and paste the following:

Kazunari Matsuda (2011). Exciton Dephasing in a Single Carbon Nanotube Studied by Photoluminescence Spectroscopy, Electronic Properties of Carbon Nanotubes, Prof. Jose Mauricio Marulanda (Ed.), ISBN: 978953-307-499-3, InTech, Available from: http://www.intechopen.com/books/electronic-properties-of-carbonnanotubes/exciton-dephasing-in-a-single-carbon-nanotube-studied-by-photoluminescence-spectroscopy

\section{INTECH}

open science | open minds

\section{InTech Europe}

University Campus STeP Ri

Slavka Krautzeka 83/A

51000 Rijeka, Croatia

Phone: +385 (51) 770447

Fax: +385 (51) 686166

www.intechopen.com

\section{InTech China}

Unit 405, Office Block, Hotel Equatorial Shanghai

No.65, Yan An Road (West), Shanghai, 200040, China

中国上海市延安西路65号上海国际贵都大饭店办公楼 405 单元

Phone: +86-21-62489820

Fax: $+86-21-62489821$ 
(C) 2011 The Author(s). Licensee IntechOpen. This chapter is distributed under the terms of the Creative Commons Attribution-NonCommercialShareAlike-3.0 License, which permits use, distribution and reproduction for non-commercial purposes, provided the original is properly cited and derivative works building on this content are distributed under the same license. 